OPEN ACCESS

Edited by:

Yonghao Zhan,

Zhengzhou University, China

Reviewed by:

Congcong Cao,

Peking University Shenzhen Hospital,

China

Yuantao Zhang,

The Chinese University of Hong Kong,

China

*Correspondence:

Zhe Shen

doctorshenzhe@163.com

Bin Yan

08timmy@163.comand

${ }^{\dagger}$ These authors have contributed equally to this work and share first

authorship

Specialty section:

This article was submitted to

Molecular Diagnostics

and Therapeutics,

a section of the journal

Frontiers in Molecular Biosciences

Received: 01 March 2021

Accepted: 22 March 2021

Published: 15 April 2021

Citation:

Lan T, Zheng Y-c, Li N-d,

Chen $X$-s, Shen $Z$ and Yan B (2021) CRISPR/dCas9-Mediated Parkin

Inhibition Impairs Mitophagy and Aggravates Apoptosis of Rat Nucleus Pulposus Cells Under

Oxidative Stress.

Front. Mol. Biosci. 8:674632. doi: 10.3389/fmolb.2021.674632

\section{CRISPR/dCas9-Mediated Parkin Inhibition Impairs Mitophagy and Aggravates Apoptosis of Rat Nucleus Pulposus Cells Under Oxidative Stress}

\author{
Tao Lan ${ }^{1 t}$, Yu-chen Zheng ${ }^{1 \dagger}$, Ning-dao $\mathrm{Li}^{2+}$, Xiao-sheng Chen ${ }^{1}$, Zhe Shen ${ }^{1 *}$ and Bin Yan ${ }^{1 *}$ \\ 1 Department of Spine Surgery, Shenzhen Second People's Hospital, The First Affiliated Hospital of Shenzhen University, \\ Shenzhen, China, ${ }^{2}$ Department of Orthopedic Surgery, Shenzhen Luohu People's Hospital, The Third Affiliated Hospital \\ of Shenzhen University, Shenzhen, China
}

Objective: The aim of this study is to explore the role of Parkin in intervertebral disk degeneration (IDD) and its mitophagy regulation mechanism.

Study design and methods: Rat nucleus pulposus (NP) cells were stimulated with hydrogen peroxide $\left(\mathrm{H}_{2} \mathrm{O}_{2}\right)$ to a mimic pathological condition. Apoptosis and mitophagy were assessed by Western blot, terminal deoxynucleotidyl transferase dUTP nick end labeling (TUNEL) assay, and immunofluorescence staining. The CRISPR-dCas9-KRAB system was used to silence the expression of Parkin.

Result: In this study, we found that Parkin was downregulated in rat NP cells under oxidative stress. In addition, treatment with $\mathrm{H}_{2} \mathrm{O}_{2}$ resulted in mitochondrial dysfunction, autophagy inhibition, and a significant increase in the rate of apoptosis of NP cells. Meanwhile, mitophagy inhibition enhanced $\mathrm{H}_{2} \mathrm{O}_{2}$-induced apoptosis. Furthermore, repression of Parkin significantly attenuated mitophagy and exacerbated apoptosis.

Conclusion: These results suggested that Parkin may play a protective role in alleviating the apoptosis of NP cells via mitophagy, and that targeting Parkin may provide a promising therapeutic strategy for the prevention of IDD.

Keywords: CRISPR/dCas9, IDD, mitophagy, apoptosis, Parkin

\section{INTRODUCTION}

Intervertebral disk degeneration (IDD) is widely known as a main contributor to low back pain (LBP), which has become a global public health problem associated with decline in quality of life and heavy socioeconomic burden (Hoy et al., 2010; Foster et al., 2018). It is estimated that approximately $80 \%$ of the population suffer from neck or back pain at some point in their lives (Côté et al., 2001). As the largest avascular structure in the human body, the intervertebral disk is a complex structure that consists of superior and inferior cartilage endplates (CEP), internal jelly-like nucleus pulposus (NP), and external annulus fibrosus (AF) (Kadow et al., 2015). NP cells are responsible for the synthesis of the extracellular matrix (ECM) and play an important role in 
maintaining the biological function of the intervertebral disk. Emerging pieces of evidence reveal that excessive apoptosis of NP cells can trigger IDD (Liu et al., 2013; Chen et al., 2016, 2017). Hence, a better understanding of the apoptosis mechanism may provide a potential therapeutic target for the prevention and treatment of IDD.

Oxidative stress is a common pathological process that is characterized by overproduction of reactive oxygen species (ROS) (Feng et al., 2017). As a main intracellular ROS-generating organelle, mitochondria are also the primary target of ROS. ROS overproduction causes mitochondrial injury. Furthermore, mitochondrial dysfunction enhances ROS generation with a positive feedback loop. Accumulated research has indicated that ROS are a potent pro-apoptotic factor for NP cells (Xia et al., 2019; Xiang et al., 2020; Zhao et al., 2020). Additionally, mitochondria are responsible for the generation of ATP, which is essential for maintaining cell survival and physiological function. Considering that mitochondrial dysfunction is implicated in the senescence and apoptosis of NP cells, mitochondrial homeostasis is vital for the health of intervertebral disks.

Mitophagy is a special type of autophagy that selectively targets damaged or redundant mitochondria to the lysosome for elimination, which is a crucial step in mitochondrial quality control (Novak and Dikic, 2011; Sun et al., 2020). It is acknowledged that mitophagy impairment results in the accumulation of defective organelles and ROS overproduction, and subsequently increases the rate of apoptosis of NP cells (Chen et al., 2020; Kang et al., 2020a,b). Hence, balance between mitophagy and apoptosis determines the fate of NP cells. However, the relationship between mitophagy and apoptosis within disk cells in response to oxidative stress remains poorly understood. Parkin is a key player in the induction of mitophagy. It regulates ubiquitination of mitochondrial outer membrane proteins and promotes degradation of dysfunctional mitochondria. It is reported that Parkin is closely related to the crosstalk between mitophagy and apoptosis in NP cells (Zhang et al., 2018; Huang et al., 2020; Madhu et al., 2020).

In this study, we hypothesized that mitophagy plays a cytoprotective role in response to the oxidative stress of NP cells. To confirm this hypothesis, hydrogen peroxide $\left(\mathrm{H}_{2} \mathrm{O}_{2}\right)$ was used to induce oxidative stress, which could mimic the pathological mechanisms of mitochondrial dysfunction and apoptosis in NP cells. Furthermore, we investigated the relationship between mitophagy and apoptosis, and the Parkin signaling pathways involved in their interactions. Finally, our study revealed that Parkin is involved in the pathogenesis of IDD and may serve as a therapeutic target for IDD.

\section{MATERIALS AND METHODS}

\section{Cell Isolation and Culture}

Nucleus pulposus cells were extracted from healthy NP of young Sprague-Dawley rats. NP tissues were isolated under a dissecting microscope and digested in $0.2 \%$ type II collagenase for approximately $4 \mathrm{~h}$ at $37^{\circ} \mathrm{C}$. The isolated cells were cultured in Dulbecco's modified Eagle medium (DMEM) and 10\% fetal bovine serum (FBS) supplemented with antibiotics (Gibco, Carlsbad, CA, United States). Second-generation NP cells were used throughout the experiments.

\section{Cell Viability Assay and NP Cells Treatment}

Cell Counting Kit-8 (CCK-8) assaying was performed to detect the viability of NP cells (CCK-8; Dojindo Co., Kumamoto, Japan) according to the protocol of the manufacturer. NP cells were seeded in 96-well plates and incubated in DMEM/F12 with $10 \%$ FBS and $1 \%$ antibiotics at $37^{\circ} \mathrm{C}$ for $24 \mathrm{~h}$. NP cells were treated using $\mathrm{H}_{2} \mathrm{O}_{2}$ with different concentrations $(0.1,0.25,0.5$, and $1 \mathrm{mM})$ for $24 \mathrm{~h}$ or $1 \mathrm{mM}$ for various times $(0,6,12$, and $24 \mathrm{~h})$. The cells were then washed with phosphate-buffered saline (PBS), and a $10 \mu \mathrm{l}$ CCK-8 solution was added to each well. The wells were incubated at $37^{\circ} \mathrm{C}$ for $1 \mathrm{~h}$. Finally, the absorbance of the wells was then measured at $450 \mathrm{~nm}$ using a micro-plate reader (BioTek, Winooski, VT, United States).

\section{Quantitative Real-Time PCR (RT-qPCR)}

Total RNA was extracted from the cultured cells using a TRIzol reagent (Invitrogen, Carlsbad, CA, United States). Reverse transcription and gene amplification procedures were conducted according to the instructions of the kit manufacturer (TransGen Biotech, Beijing, China). Glyceraldehyde 3-phosphate dehydrogenase (GAPDH) was used to normalize the gene expression of other mRNAs. PCR primers were as follows.

\section{Western Blotting}

The cells were lysed using radioimmunoprecipitation assay (RIPA) buffer (Sigma, St. Louis, MO, United States). Total protein extracts from NP cells were obtained through wholecell lysis assaying (KeyGen). Protein concentration was determined using the bicinchoninic acid (BCA) method. Protein samples were separated using sodium dodecyl sulfate-polyacrylamide gel electrophoresis (SDS-PAGE) and transferred to a polyvinylidene fluoride (PVDF) membrane. The membrane was incubated with primary antibodies against P62 (1:1,000, ab56416 Abcam, Cambridge, United Kingdom), Parkin (1:1,000, \#4211; CST, Danvers, MA, United States), LC3 (1:1,000, \#83506, CST, Danvers, MA, United States), Bcl-2 (1:1,000, \#3498; CST, Danvers, MA, United States), Bax (1:1,000, \#14796; CST, Danvers, MA, United States), GAPDH (1:10,000, \#60004-1-Ig: Proteintech) overnight at $4^{\circ} \mathrm{C}$; and target protein bands and internal reference bands were visualized and calculated using the ImageJ software (ImageJ $1.48 \mathrm{v}$, United States).

\section{Immunofluorescence}

Nucleus pulposus cells were fixed with $4 \%$ paraformaldehyde for $15 \mathrm{~min}$ and permeabilized with $0.5 \%$ Triton X-100 for $30 \mathrm{~min}$. After blocking with 5\% bovine serum albumin (BSA) for 30 min, slides were incubated with primary antibodies against P62 (1:200) overnight at $4^{\circ} \mathrm{C}$. Secondary antibody (1:100; Invitrogen, 

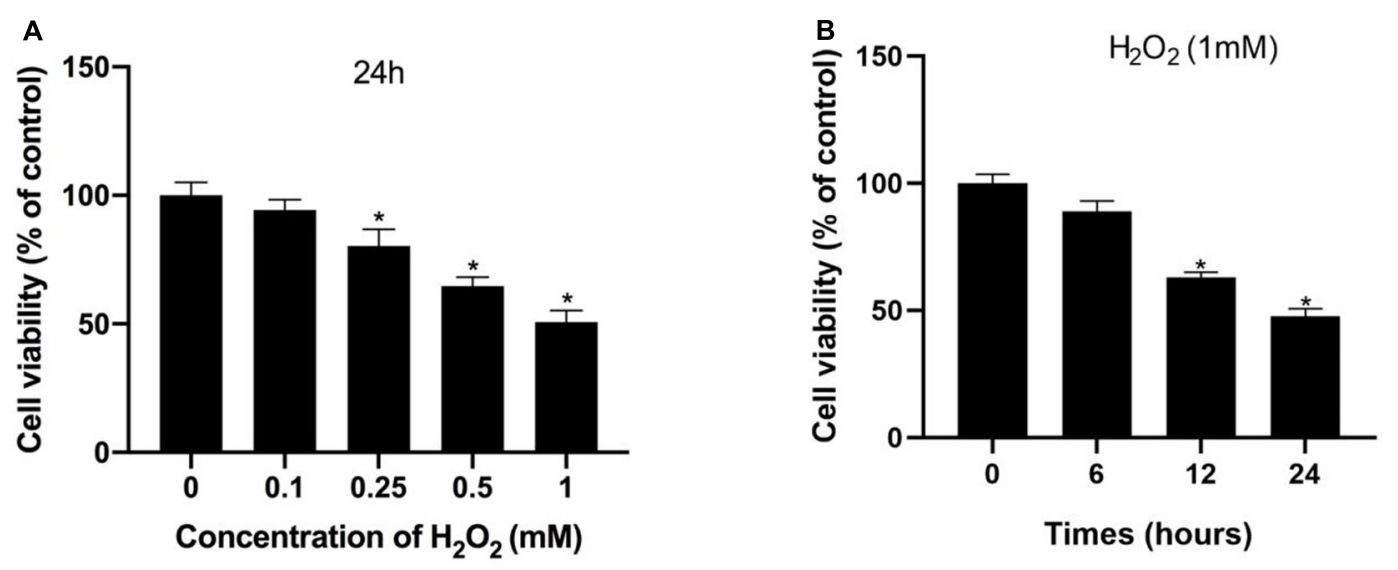

FIGURE 1 | Hydrogen peroxide inhibited cell viability in NP cells. (A,B) Effects of $\mathrm{H}_{2} \mathrm{O}_{2}$ on cell viability were detected using the Cell Counting Kit-8 (CCK-8). ${ }^{\star} p<$ 0.05 vs. control group.

Carlsbad, CA, United States) was added the next day. The nuclei were stained with $4^{\prime}, 6$-diamino-2-phenylindole (DAPI) for $1 \mathrm{~min}$. The cells were observed using a fluorescence microscope (CTR4000B, Leica, Wetzlar, Germany).

\section{Mitochondrial Membrane Potential Measurement, ATP, and Complex III}

The mitochondrial membrane potential was determined by JC-1 staining (Beyotime, Shanghai, China) according to the protocol of the manufacturer. Intracellular ATP and complex III levels were determined using the ATP Assay and ROS Assay Kits according to the instructions of the manufacturer (Beyotime, Shanghai, China).

\section{Flow Cytometry Assay of Apoptosis}

The apoptosis rate of the NF cells was detected using the Annexin V-FITC/PI Double-Staining Kit (Fushen, Shanghai, China). NP cells were collected and washed with PBS and subsequently suspended in $100 \mu \mathrm{l}$ binding buffer. The cells were then incubated with $5 \mu \mathrm{l}$ Annexin V-FITC and $5 \mu \mathrm{l} \mathrm{PI}$ at $37^{\circ} \mathrm{C}$ for $30 \mathrm{~min}$. The early (Annexin $\mathrm{V}+/ \mathrm{PI}-$ ) and the late apoptotic (Annexin $\mathrm{V}+/ \mathrm{PI}-$ ) cells were used to calculate apoptosis rate.

\section{Transfection of Plasmids}

Nucleus pulposus cells were plated using six-well plates overnight before transfection. When the cell density reached 70\% confluence, the NP cells were co-transfected with sgRNA and dcas9-KRAB plasmids using Lipofectamine 2000 (Invitrogen, Carlsbad, CA, United States) according to the protocol of the manufacture.

\section{Statistical Analysis}

Results were presented as the mean \pm SD and analyzed using SPSS 22 (IBM Corp., Armonk, NY, United States). Differences between groups were analyzed by Student's $t$-test or one-way analysis of variance (ANOVA) followed by Tukey's test. A $p$ value of less than 0.05 was considered significant.

\section{RESULTS}

\section{Hydrogen Peroxide Increases Rate of Apoptosis and Inhibits Mitophagy in Rat NP}

Initially, $\mathrm{H}_{2} \mathrm{O}_{2}$ was used to mimic oxidative stress in vitro, and CCK-8 assaying was performed to investigate the cytotoxicity of $\mathrm{H}_{2} \mathrm{O}_{2}$ in NP cells. As shown in Figures 1A,B, $\mathrm{H}_{2} \mathrm{O}_{2}$ treatment was observed to reduce cell viability in a dose- and timedependent manner. Exposure to $\mathrm{H}_{2} \mathrm{O}_{2}$ with a concentration exceeding $1 \mathrm{mmol} / \mathrm{L}$ for $24 \mathrm{~h}$ and treatment with $\mathrm{H}_{2} \mathrm{O}_{2}$ $(1 \mathrm{mmol} / \mathrm{L})$ for $24 \mathrm{~h}$ or longer showed a marked reduction in cell viability. Therefore, $\mathrm{H}_{2} \mathrm{O}_{2}$ with a concentration of $1 \mathrm{mmol} / \mathrm{L}$ was used in the subsequent experiments. Then, we assessed the apoptosis response of the NP cells to oxidative stress. The Western blot and qPCR results showed that $\mathrm{H}_{2} \mathrm{O}_{2}$-induced oxidative stress did significantly increase cleaved-caspase 3 and Bax/bcl-2 (Figures 2A-C). Meanwhile, flow cytometry by Annexin V-FITC/PI staining showed that the percentage of apoptotic NP cells was higher in the $\mathrm{H}_{2} \mathrm{O}_{2}$-treated group than in the control group (Figures 2D,E). To investigate the effect of $\mathrm{H}_{2} \mathrm{O}_{2}$ on autophagy in the NP cells, we examined the expression of LC3-II/LC3-I and p62 by Western blot. As shown in Figures 3A,B, the ratio of LC3-II/LC3-I was decreased and the level of p62 was increased after $\mathrm{H}_{2} \mathrm{O}_{2}$ treatment for $24 \mathrm{~h}$. The immunofluorescence analysis of NP cells revealed an accumulation of $\mathrm{P} 62$ protein in response to oxidative stress (Figure 3C). Taken together, these results indicated that oxidative stress induced by $\mathrm{H}_{2} \mathrm{O}_{2}$ could promote apoptosis and inhibit autophagy in NP cells.

\section{Mitochondrial Dysfunction Is Involved in the $\mathrm{H}_{2} \mathrm{O}_{2}$-Induced Apoptosis of Rat NP}

Mitophagy is responsible for quality control of mitochondria under oxidative stress. As expected, oxidative stress inhibits mitophagy but also causes mitochondrial dysfunction. After 

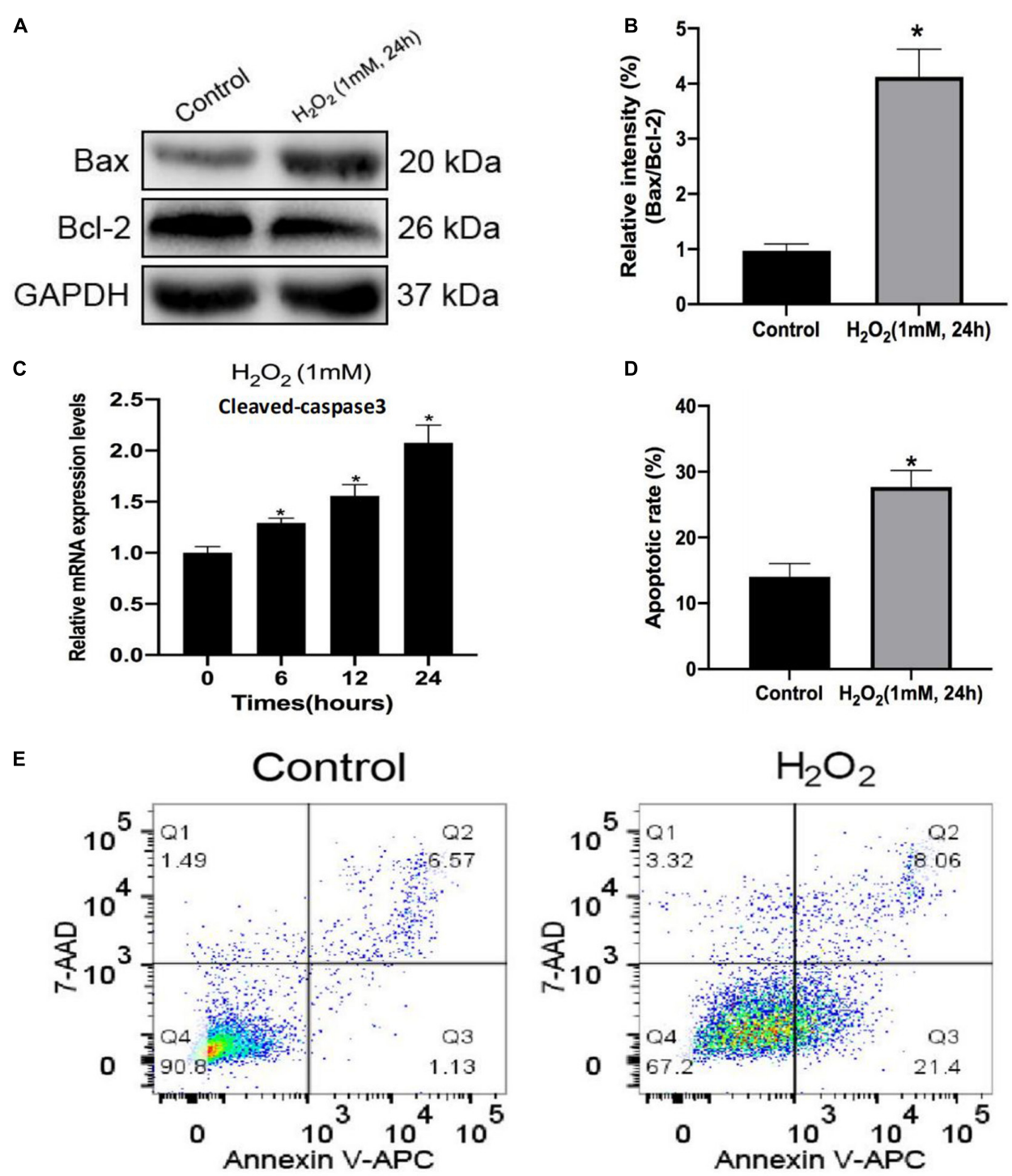

FIGURE 2 | Hydrogen peroxide promotes apoptosis in rat nucleus pulposus (NP) cells. (A,B) The protein levels of Bax and Bcl-2 in NP cells were measured by Western blotting. (C) Relative mRNA expression of cleaved caspase-3 by RT-qPCR. (D,E) Apoptosis of NP cells with or without $\mathrm{H}_{2} \mathrm{O}_{2}$ treatment was detected by flow cytometry. Data are represented as the mean \pm SD. ${ }^{*} p<0.05$ vs. control group.

treatment with $\mathrm{H}_{2} \mathrm{O}_{2}$, the green/red fluorescence ratio of NP cells significantly increased, indicating that mitochondrial membrane potential was reduced by the treatment of $\mathrm{H}_{2} \mathrm{O}_{2}$ (Figure 4A). Furthermore, the intracellular complex III level and ATP production were markedly decreased under oxidative stress (Figures 4B,C). Altogether, prolonged oxidative stress leads to mitochondrial damage.

\section{Mitophagy Inhibition by 3-MA Enhances $\mathrm{H}_{2} \mathrm{O}_{2}$-Induced Apoptosis in Rat NP}

To explore whether mitophagy was involved in the protective response against $\mathrm{H}_{2} \mathrm{O}_{2}$ induced apoptosis, we determined the expression of cleaved-caspase3, Bax, and Bcl-2. We found that NP cells pre-treated with mitophagy inhibitor 3-MA significantly increased pro-apoptotic protein expression (cleaved-caspase 3 and Bax) and decreased anti-apoptotic protein (Bcl-2) expression (Figures 5A,B). Similarly, TUNEL assay results also showed that the mitophagy inhibitor dramatically exacerbated the apoptosis of NP cells (Figure 5C,D). Collectively, these findings showed that under oxidative stress mitophagy protected rat NP cells against apoptosis.

\section{Inhibition of Parkin by dCas9-KRAB Significantly Attenuates $\mathrm{H}_{2} \mathrm{O}_{2}$-Induced Mitophagy}

In order to investigate the relationship between Parkin and IDD in rat NP cells, we determined Parkin expression under oxidative stress by Western blotting, and we found that the Parkin expression decreased significantly in the $\mathrm{H}_{2} \mathrm{O}_{2}$ group compared with that in the control group (Figure 6B). To further verify 

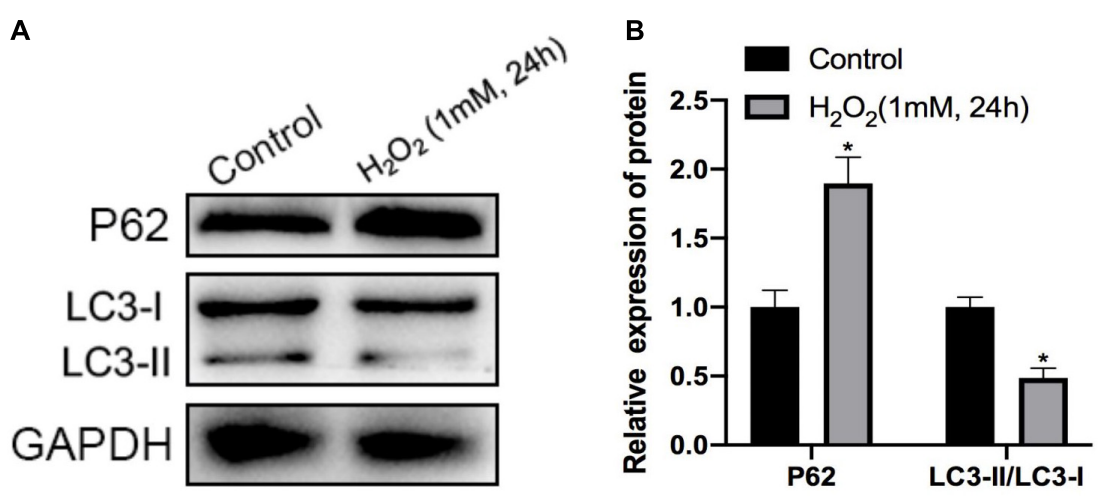

c

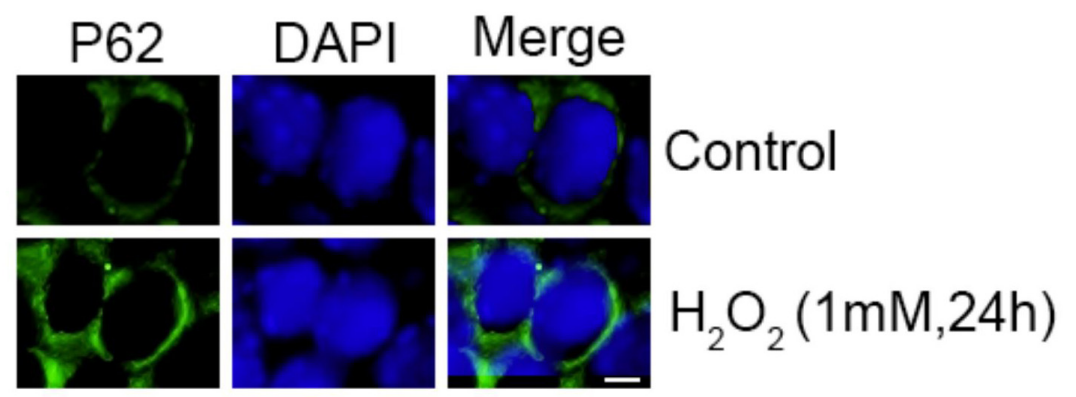

FIGURE 3 | Effects of $\mathrm{H}_{2} \mathrm{O}_{2}$ on mitophagy in NP cells. (A,B) The protein level of P62, LC3-I, and LC3-II in the NP cells was measured by Western blotting. (C) Immunofluorescence of the P62 protein in NP cells (Green signal represents P62, blue signal represents DAPI). ${ }^{*} p<0.05$ vs. control group.
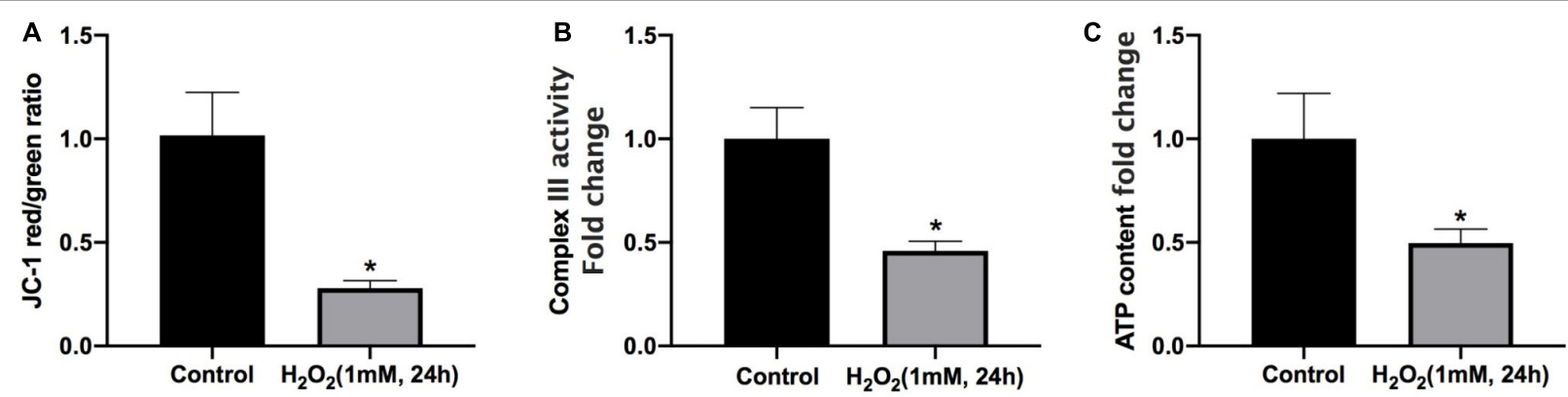

FIGURE 4 | Mitochondrial dysfunction is involved in $\mathrm{H}_{2} \mathrm{O}_{2}$-induced apoptosis of rat NP. (A) Effect of $\mathrm{H}_{2} \mathrm{O}_{2}$ on mitochondrial membrane potential. (B) Summary data for the effect of $\mathrm{H}_{2} \mathrm{O}_{2}$ on the relative content of complex III. (C) Intracellular ATP levels in NP cells. ${ }^{*} p<0.05$ vs. control group.

the role of Parkin in mitophagy, we used the CRISPR/dCas9$\mathrm{KRAB}$ system to repress the expression of Parkin (Figure 6A). We found that mitophagy decreased markedly (Figures 6C,D) while apoptosis of NP cells increased dramatically (Figures 6E,F) after repression of Parkin mediated with CRISPR/dCas9-KRAB. Overall, these results suggested that the activation of mitophagy against apoptosis was dependent on Parkin.

\section{DISCUSSION}

Intervertebral disk degeneration is a common reason for LBP, which is one of the leading causes of chronic disability. To date, conventional treatments including physical therapy, anti-inflammatory medications, and surgeries remain unsatisfactory (Legrand et al., 2007). Therefore, development of biological therapies to prevent IDD is an issue that needs to be solved urgently. Studies have shown that excessive apoptosis of NP cells plays a crucial role in the development of IDD (Guo et al., 2018; Yu et al., 2018). In addition, previous studies have shown that oxidative stress participates in the apoptosis process of NP cells (Nan et al., 2020). Nevertheless, the underlying mechanisms of apoptosis induced by oxidative stress have not been fully clarified.

In this study, we confirmed that both apoptosis and autophagy were involved in the pathogenesis of oxidative stressinduced IDD. In addition, we found that oxidative stress resulted in mitochondrial dysfunction, autophagy inhibition, 
A

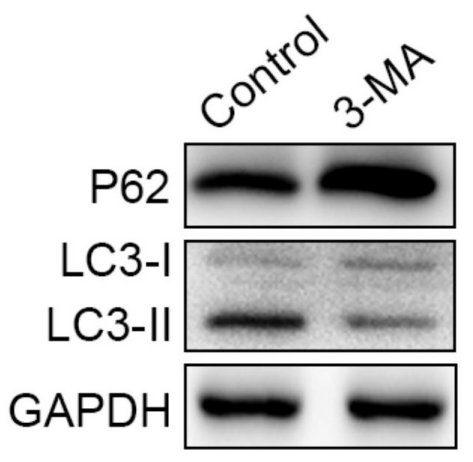

C

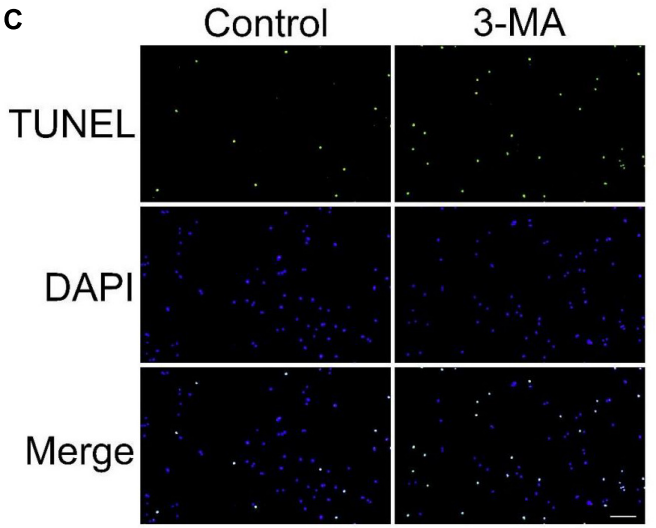

B

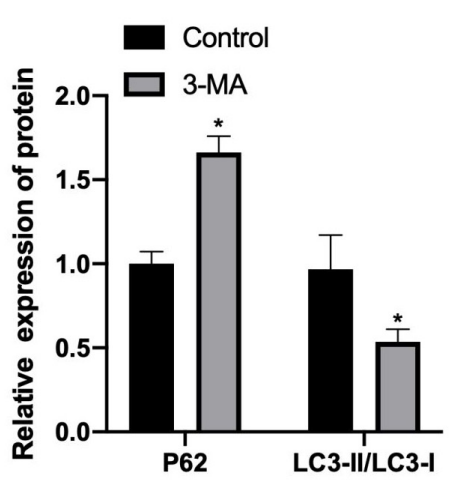

D

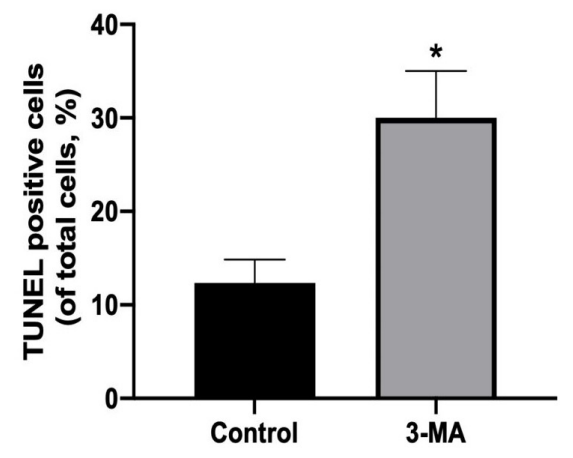

FIGURE 5 | Mitophagy inhibition enhances $\mathrm{H}_{2} \mathrm{O}_{2}$-induced apoptosis in rat NP. (A,B) Western blot of P62, LC3-I, and LC3-II expression in NP cells after treatment with 3-MA. (C,D) Representative images of TUNEL staining and quantitative analysis showing NP cells apoptosis after treatment with $3-M A$. ${ }^{*} p<0.05$ vs. control group.

and a significant increase in the rate of apoptosis of NP cells. Furthermore, we investigated the relationship between autophagy and apoptosis under oxidative stress by repressing autophagy and found that apoptosis of NP cells was dramatically enhanced, indicating that autophagy played a protective role against apoptosis in response to oxidative stress. Finally, mechanism study revealed that the expression of Parkin was suppressed after $\mathrm{H}_{2} \mathrm{O}_{2}$ treatment, and downregulation of Parkin by dCas9-KRAB led to aggravation of NP cell apoptosis and autophagy inhibition. Hence, we propose that the activation of Parkin could prevent oxidative stress-induced apoptosis and mitochondrial dysfunction in rat NP cells via the promotion of mitophagy (Figure 7).

Apoptosis of NP cells is thought to play a critical role in the progression of IDD. In mammals, there are two main apoptotic pathways: the extrinsic (also called death receptor) and the intrinsic (also called mitochondrial) (Wajant, 2002). It is acknowledged that oxidative stress-induced mitochondrial dysfunction is one of the important mechanisms that trigger the intrinsic pathway of apoptosis in NP cells (Lin et al., 2020). An oxidative injury leads to decrease in mitochondrial membrane potential and ATP production, and increase in mitochondrial outer membrane permeability, followed by the release of cytochrome $\mathrm{c}$ and activation of caspase cascade, which eventually leads to apoptosis (Chen et al., 2003). Exposure to hydrogen peroxide ( $1 \mathrm{mmol} / \mathrm{L}$ for $24 \mathrm{~h}$ ) elicited oxidative stress, and we confirmed that both mitochondria injury and apoptosis of NP cells increased markedly after $\mathrm{H}_{2} \mathrm{O}_{2}$ treatment. The results show that oxidative stress causes mitochondrialmediated intrinsic apoptosis of NP cells. Therefore, maintenance of mitochondrial function and structural integrity is the key to prevent NP cell apoptosis and IDD development.

In recent years, mitophagy has attracted increasing research interest because of its important role in the clearance of damaged mitochondria. Numerous research studies have shown that proper activation of mitophagy plays a cytoprotective role against apoptosis while excessive mitophagy leads to apoptosis (Park and Park, 2013; Lan et al., 2021). A great effort has been made to clarify the relationship between mitophagy and apoptosis, however, the role of mitophagy in ROS-induced apoptosis of NP cells remains unclear. In this study, we used 3-MA to inhibit mitophagy and found that the apoptosis rate of NP cells increased significantly, indicating that mitophagy might be the cellular self-defense mechanism for oxidative stress damage. The results are consistent with those of some previous studies (Wang J. et al., 2018; Wang Y. et al., 2018). For example, the study conducted by Lin et al. (2020) showed that mitophagy suppressed NP cells apoptosis under oxidative stress and attenuated IDD. However, opposite conclusions have also been reported in other studies. For example, Zhan et al. (2020) found that autophagy 

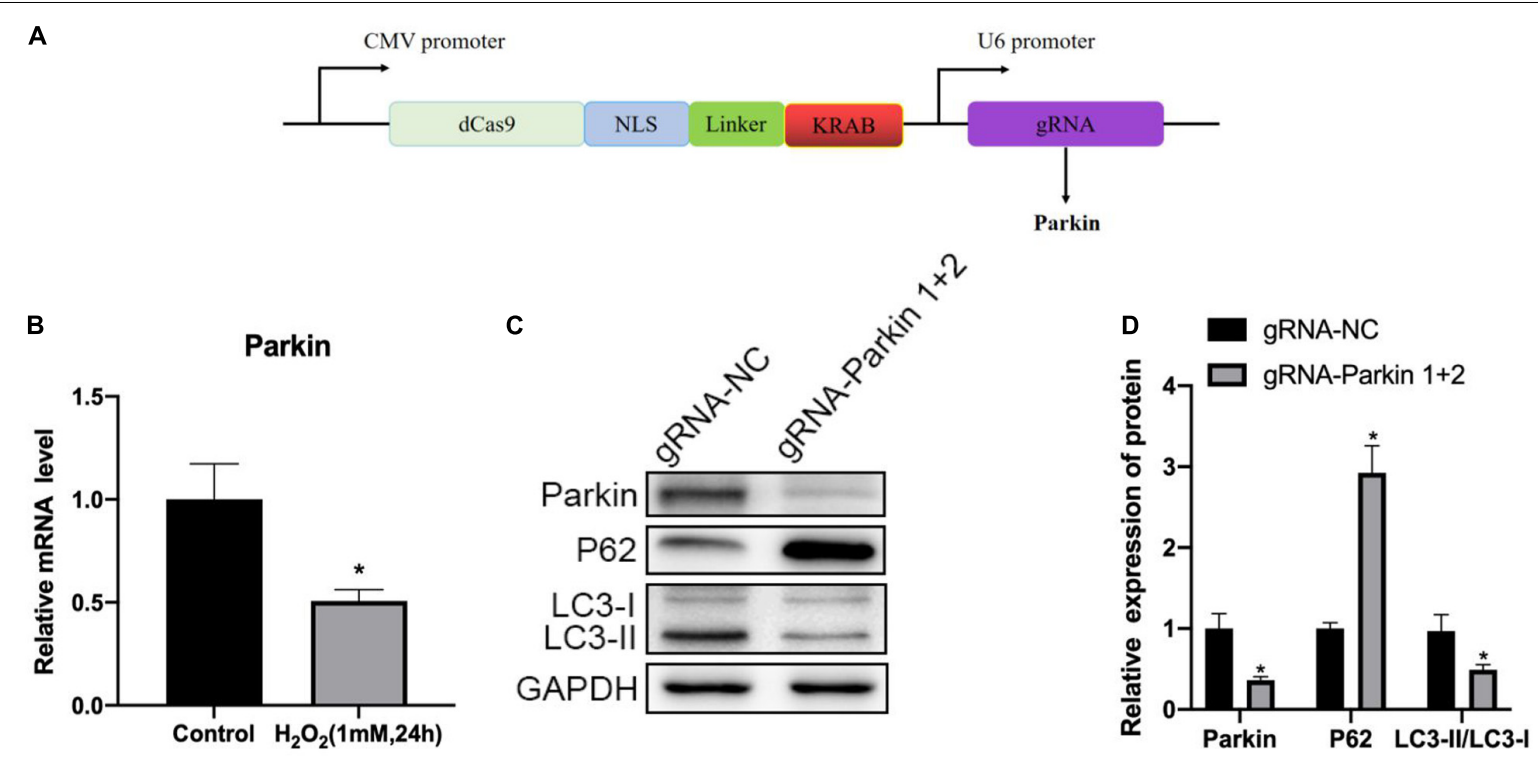

E
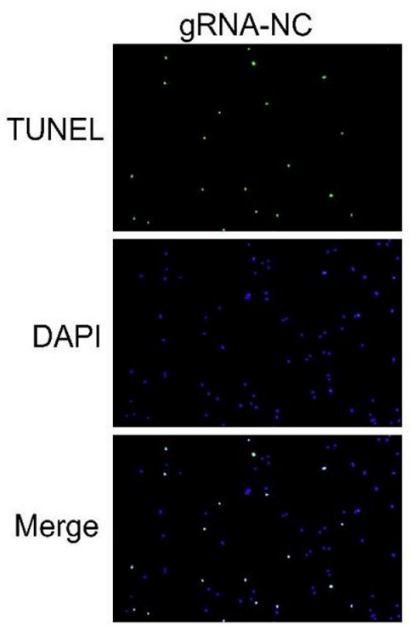

gRNA-Parkin 1+2
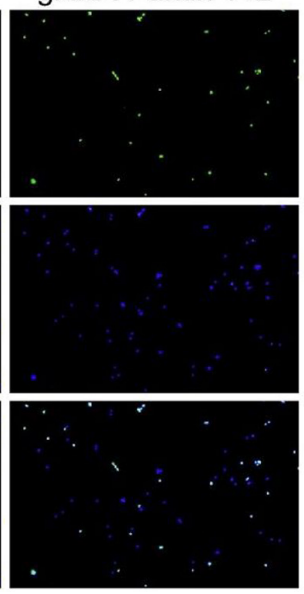

F

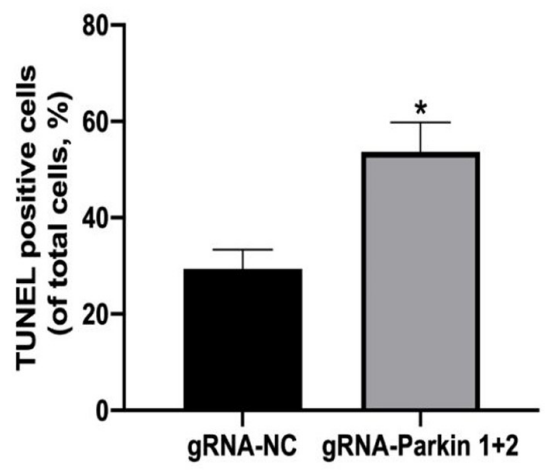

FIGURE 6 | Inhibition of Parkin by the dCas9-KRAB system significantly attenuates $\mathrm{H}_{2} \mathrm{O}_{2}$-induced mitophagy. (A) Vector map of clustered regularly interspaced palindromic repeat epigenome editing vectors targeting the Parkin gene promotor region. (B) Expression level of Parkin in NP cells. (C,D) Western blot of Parkin, P62, LC3-I, and LC3-II expression in NP cells after Parkin inhibition. (E,F) Representative images of TUNEL staining and quantitative analysis showing NP cells apoptosis after Parkin inhibition. ${ }^{*} p<0.05$ vs. control group.

activation could promote NP cell apoptosis, senescence, and ECM catabolism. Nevertheless, more and more studies show that basal-level autophagy plays a housekeeping and cytoprotective role; while autophagy dysfunction, either inadequate or excessive autophagy, leads to the death of cells. In addition, the pro-survival or pro-death effect of autophagy is also attributed to cell types, time, and degree of stimulus.

Parkin-mediated mitochondrial autophagy is the most intensively investigated mitochondrial autophagy pathway. The autophagy mechanism is translocation of Parkin to defective mitochondria and recruitment of p62/SQSTM1, followed by engulfment of damaged mitochondria by autophagosomes, and degradation by lysosomes (Nguyen et al., 2016). Parkin, as a protective protein, plays many beneficial roles in preventing degenerative diseases, such as Parkinson's disease (Kamienieva et al., 2021), osteoarthritis (Ansari et al., 2018), and Alzheimer's disease (Zhao et al., 2021). However, studies regarding the relationship between Parkin and IDD are limited. Our results revealed that Parkin was downregulated in NP cells under oxidative stress. Moreover, the inhibition of Parkin aggravated NP cell apoptosis, exacerbated mitochondrial dysfunction, and suppressed mitophagy activity, indicating that Parkin was able to prevent NP cell apoptosis and protect mitochondrial function through upregulation of mitophagy. Therefore, Parkin is a promising therapeutic target for the treatment of IDD in the future. As mentioned, there are many conventional methods to repress Parkin gene expression. In this study, we innovatively used the dCas9-KRAB system to inhibit the Parkin gene expression in NP cells under oxidative stress. The CRISPR/Cas9 system, which is derived from Streptococcus 


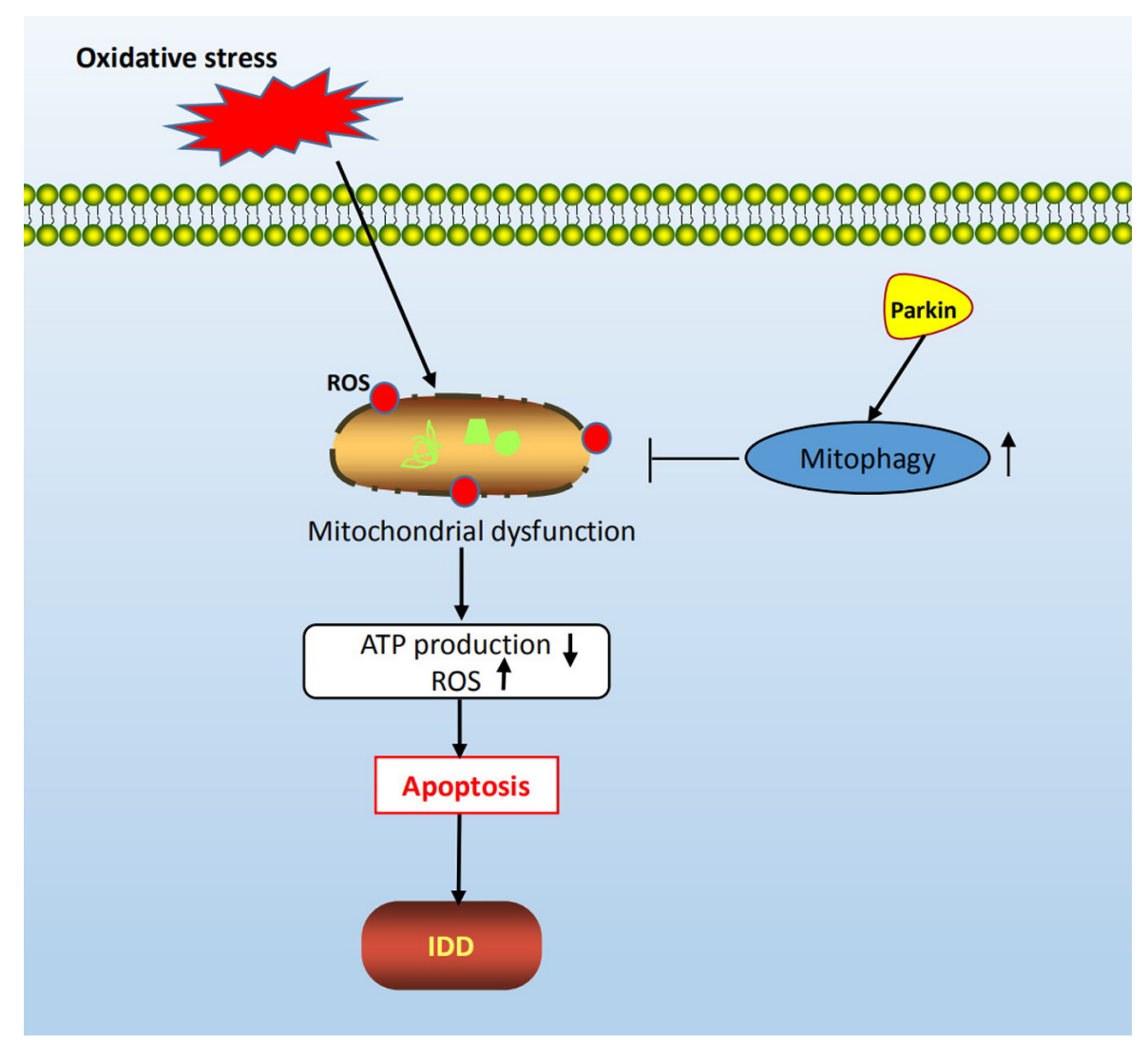

FIGURE 7 | Schematic diagram of mechanisms shows the protective role of mitophagy in rat NP cells under oxidative stress.

pyogenes, is a powerful genetic editing tool and is widely used in mammalian cells (Ran et al., 2013). CRISPR-dCas9-KRAB is a new tool to silence a target gene expression with high efficiency, low cost, and easy operation. However, the application of the CRISPR/Cas9 system in the intervertebral disk field is limited. A previous study by Stover et al. (2019) explored the potential application of CRISPR epigenome editing to target inflammatory receptors for pain modulation in degenerative intervertebral disk. Our study confirmed that the potential application of CRISPR/Cas9 as a novel gene therapy tool for IDD.

This study has several limitations. First, although our results suggest that Parkin could be a potential therapeutic target for IDD, this study is mainly in vitro; thus, further in vivo studies are needed to be carried out to provide more convincing evidence. Second, in this study we used NP cells from rats instead of NP cells from humans, and species may cause some differences. Third, since both AF cells and CEP chondrocytes also take part in the degeneration process, the response of AF cells and CEP cells to oxidative stress needs to be elucidated in the future.

\section{CONCLUSION}

In summary, this study has demonstrated that Parkin is involved in the pathogenesis of IDD and that it plays an important role in the clearance of damaged mitochondria via modulation of mitophagy. These findings suggest a potential therapeutic application of Parkin for the prevention and treatment of disk degeneration.

\section{DATA AVAILABILITY STATEMENT}

The raw data supporting the conclusions of this article will be made available by the authors, without undue reservation.

\section{ETHICS STATEMENT}

The animal study was reviewed and approved by The Animal Care and Use Committee of the Shenzhen Second People's Hospital.

\section{AUTHOR CONTRIBUTIONS}

ZS and BY conceived and designed the study. TL, YZ, and $\mathrm{N}$-DL performed the experiments and data analysis. X-SC and TL wrote and revised the manuscript. All authors contributed to the article and approved the submitted version. 


\section{REFERENCES}

Ansari, M. Y., Khan, N. M., Ahmad, I., and Haqqi, T. M. (2018). Parkin clearance of dysfunctional mitochondria regulates ROS levels and increases survival of human chondrocytes. Osteoarthr. Cartil. 26, 1087-1097. doi: 10.1016/j.joca. 2017.07.020

Chen, D., Xia, D., Pan, Z., Xu, D., Zhou, Y., Wu, Y., et al. (2016). Metformin protects against apoptosis and senescence in nucleus pulposus cells and ameliorates disc degeneration in vivo. Cell Death Dis. 7:e2441. doi: 10.1038/ cddis. 2016.334

Chen, Q., Chai, Y. C., Mazumder, S., Jiang, C., Macklis, R. M., Chisolm, G. M., et al. (2003). The late increase in intracellular free radical oxygen species during apoptosis is associated with cytochrome c release, caspase activation, and mitochondrial dysfunction. Cell Death Differ. 10, 323-334.

Chen, S., Zhao, L., Deng, X., Shi, D., Wu, F., Liang, H., et al. (2017). Mesenchymal stem cells protect nucleus pulposus cells from compression-induced apoptosis by inhibiting the mitochondrial pathway. Stem Cells Int. 2017:9843120. doi: $10.1155 / 2017 / 9843120$

Chen, Y., Lin, J., Chen, J., Huang, C., Zhang, Z., Wang, J., et al. (2020). $\mathrm{Mfn} 2$ is involved in intervertebral disc degeneration through autophagy modulation. Osteoarthr. Carti. 28, 363-374. doi: 10.1016/j.joca.2019. 12.009

Côté, P., Cassidy, J. D., and Carroll, L. (2001). The treatment of neck and low back pain: who seeks care? who goes where? Med. Care 39, 956-967.

Feng, C., Yang, M., Lan, M., Liu, C., Zhang, Y., Huang, B., et al. (2017). ROS: crucial intermediators in the pathogenesis of intervertebral disc degeneration. Oxid. Med. Cell Longev. 2017:5601593. doi: 10.1155/2017/560 1593

Foster, N. E., Anema, J. R., Cherkin, D., Chou, R., Cohen, S. P., Gross, D. P., et al. (2018). Prevention and treatment of low back pain: evidence, challenges, and promising directions. Lancet 391, 2368-2383. doi: 10.1016/s0140-6736(18) 30489-6

Guo, W., Zhang, B., Mu, K., Feng, S. Q., Dong, Z. Y., Ning, G. Z., et al. (2018). Circular RNA GRB10 as a competitive endogenous RNA regulating nucleus pulposus cells death in degenerative intervertebral disk. Cell Death Dis. 9:319. doi: 10.1038/s41419-017-0232-z

Hoy, D., Brooks, P., Blyth, F., and Buchbinder, R. (2010). The epidemiology of low back pain. Best Pract. Res. Clin. Rheumatol. 24, 769-781. doi: 10.1016/j.berh. 2010.10.002

Huang, D., Peng, Y., Li, Z., Chen, S., Deng, X., Shao, Z., et al. (2020). Compression-induced senescence of nucleus pulposus cells by promoting mitophagy activation via the PINK1/PARKIN pathway. J. Cell Mol. Med. 24, 5850-5864. doi: 10.1111/jcmm. 15256

Kadow, T., Sowa, G., Vo, N., and Kang, J. D. (2015). Molecular basis of intervertebral disc degeneration and herniations: what are the important translational questions? Clin. Orthop. Relat. Res. 473, 1903-1912. doi: 10.1007/ s11999-014-3774-8

Kamienieva, I., Duszyński, J., and Szczepanowska, J. (2021). Multitasking guardian of mitochondrial quality: parkin function and Parkinson's disease. Transl. Neurodegener. 10:5. doi: 10.1186/s40035-020-00 229-8

Kang, L., Liu, S., Li, J., Tian, Y., Xue, Y., and Liu, X. (2020a). Parkin and Nrf2 prevent oxidative stress-induced apoptosis in intervertebral endplate chondrocytes via inducing mitophagy and anti-oxidant defenses. Life Sci. 243:117244. doi: 10.1016/j.lfs.2019. 117244

Kang, L., Liu, S., Li, J., Tian, Y., Xue, Y., and Liu, X. (2020b). The mitochondriatargeted anti-oxidant MitoQ protects against intervertebral disc degeneration by ameliorating mitochondrial dysfunction and redox imbalance. Cell Prolif. 53:e12779. doi: 10.1111/cpr.12779

Lan, T., Shiyu, H., Shen, Z., Yan, B., and Chen, J. (2021). New insights into the interplay between miRNAs and autophagy in the aging of intervertebral discs. Ageing Res. Rev. 65:101227. doi: 10.1016/j.arr.2020. 101227
Legrand, E., Bouvard, B., Audran, M., Fournier, D., and Valat, J. P. (2007). Sciatica from disk herniation: medical treatment or surgery? Joint Bone Spine 74, 530-535. doi: 10.1016/j.jbspin.2007.07.004

Lin, J., Zhuge, J., Zheng, X., Wu, Y., Zhang, Z., Xu, T., et al. (2020). Urolithin A-induced mitophagy suppresses apoptosis and attenuates intervertebral disc degeneration via the AMPK signaling pathway. Free Radic. Biol. Med. 150, 109-119. doi: 10.1016/j.freeradbiomed.2020. 02.024

Liu, G., Cao, P., Chen, H., Yuan, W., Wang, J., and Tang, X. (2013). MiR-27a regulates apoptosis in nucleus pulposus cells by targeting PI3K. PLoS One 8:e75251. doi: 10.1371/journal.pone.00 75251

Madhu, V., Boneski, P. K., Silagi, E., Qiu, Y., Kurland, I., Guntur, A. R., et al. (2020). Hypoxic regulation of mitochondrial metabolism and mitophagy in nucleus pulposus cells is dependent on HIF-1 $\alpha$-BNIP3 Axis. J. Bone Miner Res. 35, 1504-1524. doi: 10.1002/jbmr.4019

Nan, L. P., Wang, F., Liu, Y., Wu, Z., Feng, X. M., Liu, J. J., et al. (2020). 6-gingerol protects nucleus pulposus-derived mesenchymal stem cells from oxidative injury by activating autophagy. World J. Stem Cells 12, 1603-1622. doi: 10.4252/wjsc.v12.i12.1603

Nguyen, T. N., Padman, B. S., and Lazarou, M. (2016). Deciphering the molecular signals of PINK1/Parkin mitophagy. Trends Cell Biol. 26, 733-744.

Novak, I., and Dikic, I. (2011). Autophagy receptors in developmental clearance of mitochondria. Autophagy 7, 301-303. doi: 10.4161/auto.7.3.14509

Park, E. Y., and Park, J. B. (2013). High glucose-induced oxidative stress promotes autophagy through mitochondrial damage in rat notochordal cells. Int. Orthop. 37, 2507-2514. doi: 10.1007/s00264-013-2037-8

Ran, F. A., Hsu, P. D., Wright, J., Agarwala, V., Scott, D. A., and Zhang, F. (2013). Genome engineering using the CRISPR-Cas9 system. Nat. Protoc. 8, 2281-2308. doi: $10.1038 /$ nprot.2013.143

Stover, J. D., Farhang, N., Lawrence, B., and Bowles, R. D. (2019). Multiplex epigenome editing of dorsal root ganglion neuron receptors abolishes redundant interleukin 6 , tumor necrosis factor alpha, and interleukin $1 \beta$ signaling by the degenerative intervertebral disc. Hum. Gene Ther. 30, 11471160. doi: $10.1089 /$ hum. 2019.032

Sun, K., Jing, X., Guo, J., Yao, X., and Guo, F. (2020). Mitophagy in degenerative joint diseases. Autophagy. doi: 10.1080/15548627.2020.1822097 [Epub ahead of print].

Wajant, H. (2002). The Fas signaling pathway: more than a paradigm. Science 296, 1635-1636. doi: 10.1126/science. 1071553

Wang, J., Nisar, M., Huang, C., Pan, X., Lin, D., Zheng, G., et al. (2018). Small molecule natural compound agonist of SIRT3 as a therapeutic target for the treatment of intervertebral disc degeneration. Exp. Mol. Med. 50, $1-14$.

Wang, Y., Shen, J., Chen, Y., Liu, H., Zhou, H., Bai, Z., et al. (2018). PINK1 protects against oxidative stress induced senescence of human nucleus pulposus cells via regulating mitophagy. Biochem. Biophys. Res. Commun. 504, 406-414. doi: 10.1016/j.bbrc.2018.06.031

Xia, C., Zeng, Z., Fang, B., Tao, M., Gu, C., Zheng, L., et al. (2019). Mesenchymal stem cell-derived exosomes ameliorate intervertebral disc degeneration via anti-oxidant and anti-inflammatory effects. Free Radic. Biol. Med. 143, $1-15$.

Xiang, Q., Cheng, Z., Wang, J., Feng, X., Hua, W., Luo, R., et al. (2020). Allicin attenuated advanced oxidation protein product-induced oxidative stress and mitochondrial apoptosis in human nucleus pulposus cells. Oxid. Med. Cell Longev. 2020:6685043. doi: 10.1155/2020/6685043

Yu, Y., Zhang, X., Li, Z., Kong, L., and Huang, Y. (2018). LncRNA HOTAIR suppresses TNF- $\alpha$ induced apoptosis of nucleus pulposus cells by regulating miR-34a/Bcl-2 axis. Biomed. Pharmacother. 107, 729-737. doi: 10.1016/j. biopha.2018.08.033

Zhan, S., Wang, K., Xiang, Q., Song, Y., Li, S., Liang, H., et al. (2020). IncRNA HOTAIR upregulates autophagy to promote apoptosis and senescence of nucleus pulposus cells. J. Cell Physiol. 235, 2195-2208. doi: 10.1002/jcp. 29129

Zhang, Z., Xu, T., Chen, J., Shao, Z., Wang, K., Yan, Y., et al. (2018). Parkinmediated mitophagy as a potential therapeutic target for intervertebral 
disc degeneration. Cell Death Dis. 9:980. doi: 10.1038/s41419-018-1 024-9

Zhao, N., Xia, J., and Xu, B. (2021). Physical exercise may exert its therapeutic influence on Alzheimer's disease through the reversal of mitochondrial dysfunction via SIRT1-FOXO1/3-PINK1-Parkin-mediated mitophagy. J. Sport Health Sci. 10, 1-3. doi: 10.1016/j.jshs.2020.08.009

Zhao, Y., Qiu, C., Wang, W., Peng, J., Cheng, X., Shangguan, Y., et al. (2020). Cortistatin protects against intervertebral disc degeneration through targeting mitochondrial ROS-dependent NLRP3 inflammasome activation. Theranostics 10, 7015-7033. doi: 10.7150/thno.45359
Conflict of Interest: The authors declare that the research was conducted in the absence of any commercial or financial relationships that could be construed as a potential conflict of interest.

Copyright (c) 2021 Lan, Zheng, Li, Chen, Shen and Yan. This is an open-access article distributed under the terms of the Creative Commons Attribution License (CC BY). The use, distribution or reproduction in other forums is permitted, provided the original author(s) and the copyright owner(s) are credited and that the original publication in this journal is cited, in accordance with accepted academic practice. No use, distribution or reproduction is permitted which does not comply with these terms. 